\title{
The First Case of Ochrobactrum pseudogrignonense Bacteremia in Korea
}

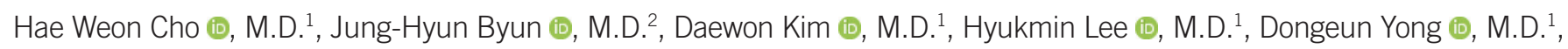
and Kyung Won Lee $\mathbb{0}$, M.D. ${ }^{1}$

${ }^{1}$ Department of Laboratory Medicine, Yonsei University College of Medicine, Seoul, Korea; ${ }^{2}$ Department of Laboratory Medicine, Gyeongsang National University Hospital Gyeongsang National University College of Medicine, Jinju, Korea

\section{Dear Editor,}

Ochrobactrum pseudogrignonense is a gram-negative, nonspore-forming, aerobic bacillus, rarely encountered in clinical specimens. It was originally isolated in Scandinavia from the blood of a 28-year-old man in 1992 and the ear of a newborn in 2000 [1]. Since then, 0 . pseudogrignonense has been isolated from environmental sources [2-4]; however, its pathogenicity in humans has not been explored extensively. We report the first case of $O$. pseudogrignonense bacteremia in Korea-and only the third in the world [1], to our knowledge - and species identification was verified by $16 \mathrm{~S}$ rRNA and recA gene sequencing. This study was approved by the Institutional Review Board (IRB) of Yonsei University of Medicine, Seoul, Korea (2019-1369). This is a retrospective report and hence IRB approval was obtained without consent forms.

A 44-year-old man with hypertension, diabetes mellitus, and dilated cardiomyopathy presented with aggravated dyspnea and falling blood pressure and was admitted to a hospital in March 2018. After three days, the patient received extracorporeal membrane oxygenation (ECMO) and was referred to a tertiary-care hospital in Seoul for heart transplantation. After transfer, the patient's vital signs were as follows: blood pressure, 110/87 mm Hg; pulse rate, 120/minutes; respiration rate, 16/minutes; and body temperature, $36.5^{\circ} \mathrm{C}$. The initial laboratory results were as follows: hemoglobin, $175 \mathrm{~g} / \mathrm{L}$; white blood cell (WBC) count, $28.5 \times 10^{9} / \mathrm{L}$; and platelet count, $118 \times 10^{9} / \mathrm{L}$. The patient was initially placed on a vancomycin and piperacillin-tazobactam regimen. Although the patient had no fever one day after admission, his serum C-reactive protein level was $514.3 \mathrm{nmol} / \mathrm{L}$, WBC count was $42.6 \times 10^{9} / \mathrm{L}$, and delta neutrophil index was $12.4 \%$, suggesting an increased immature granulocyte fraction [5]. As fever may not be apparent in ECMO patients with bacteremia [6] and considering the deteriorated laboratory results, the antibiotic regimen was changed from piperacillin-tazobactam to meropenem.

One set of aerobic and anaerobic blood cultures from the central venous line and two sets from different peripheral venipunctures were collected before initiation of antibiotic therapy and incubated for 36 hours in the BACT/ALERT 3D blood culture system (bioMérieux, Durham, NC, USA). All three aerobic vials demonstrated bacterial growth; they formed beige, non-hemolytic, distinct small circular colonies on blood and MacConkey agar plates within 24 hours of incubation at $35^{\circ} \mathrm{C}$ (Fig. 1A \& B). Gram-staining indicated gram-negative, rod-shaped bacteria without spores (Fig. 1C). Matrix-assisted laser desorption/ionization time-of-flight mass spectrometry (MALDI-TOF MS; Bruker
Received: October 14, 2019

Revision received: November 18, 2019

Accepted: January 22, 2020

Corresponding author: Dongeun Yong, M.D.

Department of Laboratory Medicine, Research Institute of Bacterial Resistance, Yonsei University College of Medicine, 50 Yonsei-ro, Seodaemun-gu, Seoul 03722, Korea

Tel: +82-2-2228-2442, Fax: +82-2-364-1583, E-mail: deyong@yuhs.ac

\section{(c) (1) $(9$}

(C) Korean Society for Laboratory Medicine

This is an Open Access article distributed under the terms of the Creative Commons Attribution Non-Commercial License (https://creativecommons.org/licenses/by-nc/4.0) which permits unrestricted non-commercial use, distribution, and reproduction in any medium, provided the original work is properly cited. 

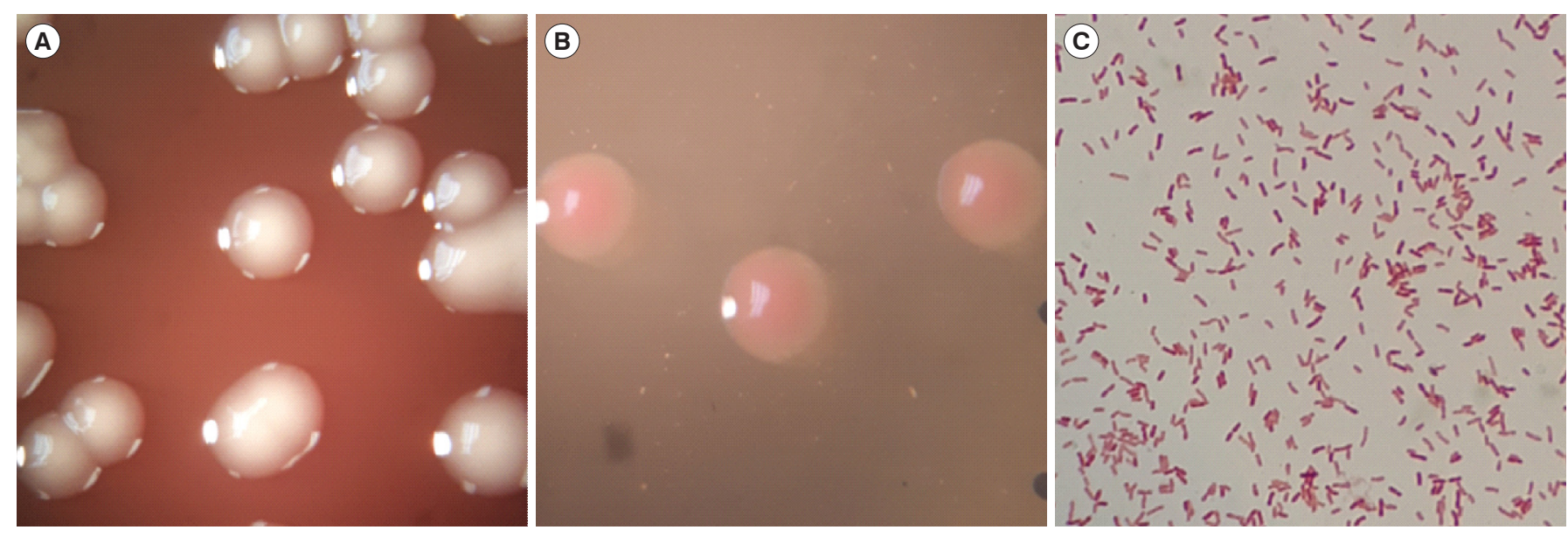

Fig. 1. Colony and microscopic morphology of the O. pseudogrignonense isolate. (A) Colonies on blood agar (10×) and (B) MacConkey agar $(10 \times)$ plates. (C) Gram staining of the isolate $(1,000 \times)$.

Daltonics, Bremen, Germany) of the isolate revealed signals characteristic of $O$. gallinifaecis with a score of 1.81 ; however, both the VITEK MS and VITEK2 systems (bioMérieux, Marcy l'Etoile, France) failed to identify the isolate.

To characterize the bacterium, molecular identification was performed by DNA amplification and sequencing of 16S rRNA [7]. Based on the EzTaxon database (http://www.ezbiocloud. net), both 373 and 1,374 bp 16S rRNA sequences of the isolate shared $100 \%$ sequence identity with those of $O$. pseudogrignonense (GenBank accession number AM422371) and 99.3\% with those of Ochrobactrum thiophenivorans (GenBank number AM490617). Based on the Ribosomal Database Project (https:// rdp.cme.msu.edu/), the isolate had a sequence match score of 1.0 for 0 . pseudogrignonense and 0.96 for 0 . thiophenivorans.

As 16S rRNA did not provide the resolution to distinguish the species [7], alternative analysis using the recA gene was performed $[8,9]$. We constructed primer pairs for partial sequencing O. pseudogrignonense (GenBank number AM422877.1) and $O$. thiophenivorans (GenBank number KF866345) recA: recA-O.pseudo-f (5'-TTCGGGTAAAACCACTCTCG-3')/recA-O. pseudo-r (5'-ATGTCGAACTCGACCTGCTT-3') and recA-0.thio-f (5'-GCAAGGGCTCAATCATGC-3')/recA-0.thio-r (5'-AATCACCCATTTCACCTTCG-3'). The isolate was amplified successfully only with the 0 . pseudogrignonense recA primer pair (510 bp). This PCR product shared $100 \%$ sequence identity with the recA gene of $O$. pseudogrignonense, as determined using the GenBank alignment search tool (BLAST; https://blast.ncbi.nIm.nih.gov/ Blast.cgi).

We performed antimicrobial susceptibility testing (AST) of the isolate using the VITEK2 system with the AST-N225 card, which
Table 1. Antimicrobial susceptibilities of the $O$. pseudogrignonense isolate

\begin{tabular}{lcc}
\hline Antimicrobial agents & Susceptibility & MIC $(\mu \mathrm{g} / \mathrm{mL})$ \\
\hline Amikacin & $\mathrm{S}$ & 8 \\
Ampicillin/Sulbactam & $\mathrm{S}$ & $8 / 4$ \\
Aztreonam & $\mathrm{R}$ & $\geq 64$ \\
Ceftazidime & $\mathrm{S}$ & 8 \\
Ciprofloxacin & $\mathrm{S}$ & $\leq 0.25$ \\
Colistin & $\mathrm{S}$ & $\leq 0.5$ \\
Cefepime & $\mathrm{S}$ & $\leq 1$ \\
Cefotaxime & $\mathrm{S}$ & 4 \\
Gentamicin & $\mathrm{S}$ & $\leq 1$ \\
Imipenem & $\mathrm{S}$ & 0.5 \\
Meropenem & $\mathrm{S}$ & 1 \\
Minocycline & $\mathrm{S}$ & $\leq 1$ \\
Piperacillin & $\mathrm{R}$ & $\geq 128$ \\
Piperacillin-Tazobactam & $\mathrm{R}$ & $\geq 128 / 4$ \\
Cotrimoxazole & $\mathrm{S}$ & $\leq 20$ \\
Tigecycline & $\mathrm{S}$ & $\leq 0.5$ \\
\hline Abid & &
\end{tabular}

Abbreviations: MIC, minimum inhibitory concentration; S, susceptible; R, resistant.

was interpreted based on the breakpoints for other non-Enterobacteriaceae [10]. The results showed that the isolate was susceptible to meropenem and resistant to piperacillin-tazobactam (Table 1). Consistent with the AST results, two days after meropenem treatment, the patient's WBC count decreased to 16.0 $\times 10^{\circ} / \mathrm{L}$, and blood culture results were negative for five days. The patient recovered without any evident sequelae. 
Ochrobactrum species are emerging pathogens with low virulence that can cause severe systemic infections not only in patients with underlying diseases but also in immunocompetent patients $[8,9]$. This is the first case of human infection with $O$. pseudogrignonense in Korea. As the isolate showed resistance to piperacillin-tazobactam, accurate identification and AST were critical for timely and appropriate treatment; however, it was difficult to identify the isolate using commercial bacterial identification kits and MALDI-TOF MS. Owing to the high similarity of 16S rRNA sequences among Ochrobactrum species, as well as Brucella species $[8,9]$, additional sequencing, such as of the recA gene, is needed for accurate species-level identification.

\section{ACKNOWLEDGEMENTS}

We are grateful to Myungsook Kim, Hyeon Ju Oh and the medical laboratory technologists from the department of clinical microbiology for the excellent technical assistance.

\section{AUTHOR CONTRIBUTIONS}

HWC and DY conceptualized and designed the study; searched the scientific literature; collected, analyzed, and interpreted the data; and wrote and revised the report. JHB, DK, HL, and KWL designed the study and revised the report. DY and KWL supervised the study and gave administrative, technical, and material support and secured the funding.

\section{CONFLICTS OF INTEREST}

No potential conflicts of interest relevant to this article are reported.

\section{RESEARCH FUNDING}

This work was supported by a grant of the Korea Health Technology R\&D Project through the Korea Health Industry Development Institute (KHIDI), funded by the Ministry of Health \& Welfare, Republic of Korea (grant number: HI14C1324).

\section{ORCID}

Hae Weon Cho

https://orcid.org/0000-0002-3017-5026

Jung-Hyun Byun https://orcid.org/0000-0001-5909-5807

Daewon Kim https://orcid.org/0000-0002-3487-5943

Hyukmin Lee

Dongeun Yong

Kyungwon Lee

https://orcid.org/0000-0002-8523-4126

https://orcid.org/0000-0002-1225-8477

https://orcid.org/0000-0003-3788-2134

\section{REFERENCES}

1. Kampfer P, Scholz HC, Huber B, Falsen E, Busse HJ. Ochrobactrum haematophilum sp. nov. and Ochrobactrum pseudogrignonense sp. nov., isolated from human clinical specimens. Int J Syst Evol Microbio 2007; 57:2513-8.

2. Yang Y, Yu X, Zhang R. Draft genome sequence of Ochrobactrum pseudogrignonense strain CDB2, a highly efficient arsenate-resistant soil bacterium from arsenic-contaminated cattle dip sites. Genome Announc 2013; $1: \mathrm{e} 00173-13$

3. Zhao G, Ma F, Wei L, Chua H, Chang CC, Zhang XJ. Electricity generation from cattle dung using microbial fuel cell technology during anaerobic acidogenesis and the development of microbial populations. Waste Manag 2012;32:1651-8.

4. Wu Z, Peng W, He X, Wang B, Gan B, Zhang X. Mushroom tumor: a new disease on Flammulina velutipes caused by Ochrobactrum pseudogrignonense. FEMS Microbiol Lett 2016;363:fnv226.

5. Seok Y, Choi JR, Kim J, Kim YK, Lee J, Song J, et al. Delta neutrophi index: a promising diagnostic and prognostic marker for sepsis. Shock 2012;37:242-6.

6. Burket JS, Bartlett RH, Vander Hyde K, Chenoweth CE. Nosocomial infections in adult patients undergoing extracorporeal membrane oxygenation. Clin Infect Dis 1999;28:828-33.

7. CLSI. Interpretive criteria for identification of bacteria and fungi by targeted DNA sequencing. 2nd ed. MM18. Wayne, PA: Clinical and Laboratory Standards Institute. 2018.

8. Scholz HC, Al Dahouk S, Tomaso H, Neubauer H, Witte A, Schloter M, et al. Genetic diversity and phylogenetic relationships of bacteria belonging to the Ochrobactrum-Brucella group by recA and 16S rRNA genebased comparative sequence analysis. Syst Appl Microbiol 2008;31:116.

9. Scholz HC, Tomaso H, Al Dahouk S, Witte A, Schloter M, Kampfer P, et al. Genotyping of Ochrobactrum anthropi by recA-based comparative sequence, PCR-RFLP, and 16S rRNA gene analysis. FEMS Microbio Lett 2006;257:7-16.

10. CLSI. Performance standards for antimicrobial susceptibility testing. 28th ed. M100. Wayne, PA: Clinical and Laboratory Standards Institute. 2018. 